\title{
The evaluation of the grape polyphenoles nootropic activity in resveratrol and in the food concentrate "Enoant"
}

\author{
Natalia Tribrat $^{1 *}$, Yulia Knizhnik ${ }^{1}$, Denis Khusainov ${ }^{1}$, Alexander Tribrat $^{2}$, Elviza \\ Dzjeldubaeva $^{1}$, Lyubov Akishina ${ }^{3}$ and Svitlana Chornobay ${ }^{4}$ \\ ${ }^{1}$ Taurida Academy, V.I. Vernadsky Crimean Federal University, 295007, Simferopol, the Republic \\ of Crimea, the Russian Federation \\ ${ }^{2}$ Medical Academy named after S.I. Georgievsky, V.I. Vernadsky Crimean Federal University, \\ 295051 Simferopol, the Republic of Crimea, Russia \\ ${ }^{3}$ Health center, Feodosia, 298112, the Republic of Crimea, Russia \\ ${ }^{4}$ V.I. Vernadsky Crimean Federal University, 295001, Simferopol, the Republic of Crimea, Russia
}

\begin{abstract}
The article is devoted to the comparison of the nootropic activity of the grape polyphenoles in resveratrol and in the food concentrate "Enoant" at doses $20 \mathrm{mg} / \mathrm{kg}$, using the Barnes maze in animals. It was found out that resveratrol and the food concentrate "Enoant" demonstrate the positive nootropic effect in the Barnes maze, which is expressed by the changes in the time spent on searching for a burrow and the number of incorrect definitions of the true burrow.
\end{abstract}

Keywords: grape polyphenols, nootropic activity, food concentrate "Enoat".

\section{Introduction}

Grape polyphenols have multiple biological effects. A special attention should be given to the nootropic effects, mediated increase of neuronal transmission, improving methabolic function and induction of neurogenesis [1]. The neuroprotective effect of grape polyphenols prevents age-related damage to the central nervous system due to their antioxidant and antiinflammatory activity. Resveratrol is recognized as a reference substance among the grapes polyphenoles. Besides, the resveratrol has a wide biological effectiveness.

It is necessary to mention that the food concentrate "Enoant" is one of the local Crimean products. This food concentrate is the derivative of the grape polyphenoles. It contains numerous polyphenoles: anthocyanins, flavonoids and non-flavonoid polyphenoles. The food concentrate "Enoant" has not been sufficiently researched. There is a little number of the relevant studies for today.

\section{Purpose of the study}

*Corresponding author : tribratnatalia@ rambler.ru 
The goal of this study is to conduct a comparative analysis of nootropic activity of polyphenoles in resveratrol and the food concentrate "Enoant".

\section{Experiment}

The study was carried out on the basis of the Laboratory for Assessing the Functional State of a Person at the Department of Human and Animal Physiology and Biophysics of Taurida Academy, V. I. Vernadsky Crimean Federal University.

For the experiments, 60 laboratory sexually mature female Wistar rats weighing from 200 to $280 \mathrm{~g}$ at the age of 88 months were selected. The animals were divided into three groups of 20 animals each. The animals of the first group is control. The animals of the second group took the supplement of resveratrol (manufacturer - Solgar) at a dose of 20 $\mathrm{mg} / \mathrm{kg}$ preorally (dissolved in water) during the period of 15 days.

The animals of the third group took the food concentrate "Enoant" at a dose of $20 \mathrm{mg} / \mathrm{kg}$ preorally (dissolved in water) during the period of 15 days.

The food concentrate of the grape polyphenoles "Enoant" is produced according to the technical conditions that normalize the content of phenolic substances in the product at the level of $18-20 \mathrm{~g} / \mathrm{dm}^{3}$, controlled photocalorimetrically using the Folin-Chocalteu reagent [TC U 00334830.018-99. The food concentrate of the grape polyphenoles "Enoant", IVIV Magarach]. The antioxidant activity of the "Enoant" concentrate exceeds the antioxidant properties of ascorbic acid by 15 times, and the antioxidant properties of blood plasma by more than 3 orders of magnitude [2-4].

The rats were preselected with an average level of emotional and motor activity in the open field test.

The testing on the Barnes maze aims at defining the strategy and assessing the cognitive abilities. The study involved pre-testing during the first two days, when the animals were placed in the center of the arena (the Barnes maze) and for a few minutes the rats quested for true burrow. The maze Barnes has only one true burrow and many false burrows. In the cases where the rats could not find the true burrow, the animal was guided carefully by the hand. The animal could stay in the true burrow for a few minutes. On the 3rd day we shot a video of the animals' behavior and search for the burrow. During the animal testing in the Barnes maze, the following indicators were assessed:

- the duration of the animals' search for the true burrow (s)

- the number of mistakes, made by animals before reaching the goal.

The subsequent registration of the investigated indicators was performed on the $5^{\text {th }}, 10^{\text {th }}$, $15^{\text {th }}$ days.

During the behavioral testing, after each animal, the arena was cleaned with liquid to eliminate the smell of the previous animal.

The study was carried out mainly during the daytime.

The animals were kept in the standardized vivarium conditions at the temperatures from 18 to $22^{\circ} \mathrm{C}$, on a Rehofix MK 2000 bedding (based on corn cobs) with natural illumination of a 12-hour light-dark cycle. There was an access to water in accordance with the State Standard 33215-2014 "Guidelines for the maintenance and care of laboratory animals. Rules for the equipment of premises and organizational procedures" and to full-fledged granulated food (the State Standard R-50258-92).

The calculations, statistical processing and graphic presentation of the data obtained in the work were carried out using Microsoft Excel and StatSoft \STATISTICA 8. Statistic data processing was carried out using the nonparametric Fridmann test and test KraskellWallis 


\section{Results and discussion}

As a result, the study has shown, the polyphenoles of grape promote the change of nootropic activity indicators - the time spent on finding the true burrow and the number of mistakes made by the rats before reaching the goal (true burrow).

The most early changes were registered in the group of rats, that took the food concentrate "Enoant" - there was a reduction in the time to find the true burrow by $50 \%$ $(\mathrm{p} \leq 0.05)$ on the $5^{\text {th }}$ day and by $55 \%(\mathrm{p} \leq 0.05)$ on the $10^{\text {th }}$ day in comparison with the data of these indicators, registered in the control group.

In the rats, that took resveratrol, the reduction in a time at a late date was registered - on $10^{\text {th }}$ day by $73 \%(\mathrm{p} \leq 0.05)$ in comparison the data, that were registered in the control group. By the $15^{\text {th }}$ day the time indicator equalized, because all the groups of animals were successfully taught.

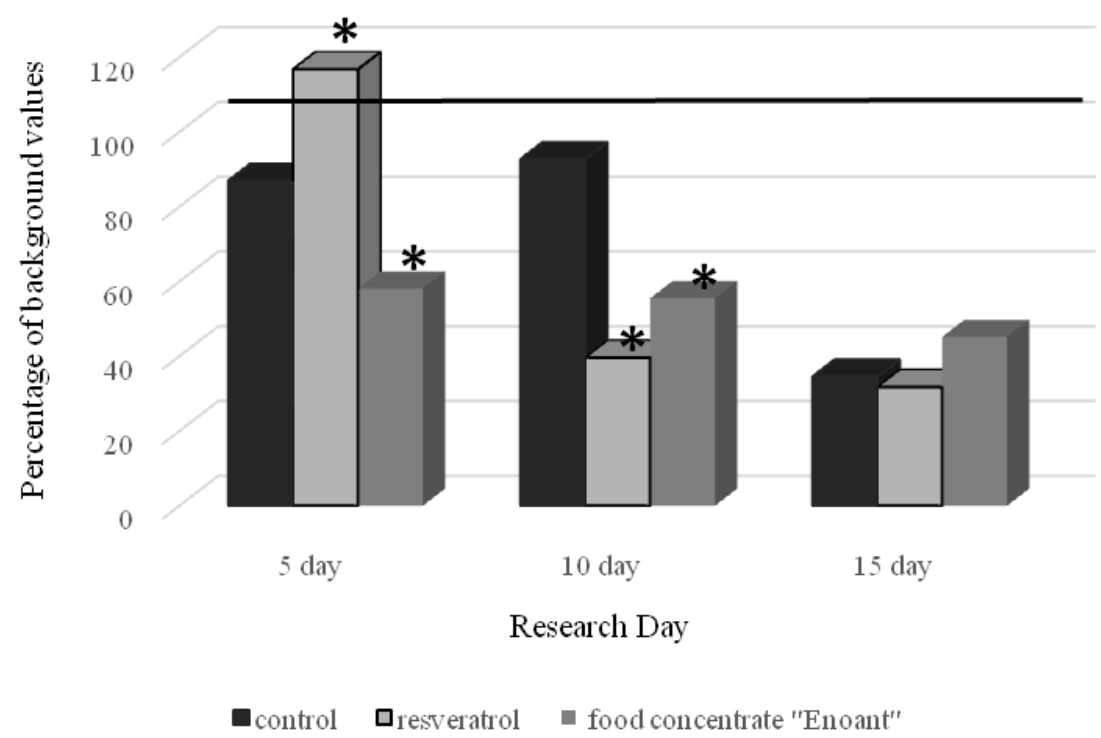

Fig. 1. The changes of the time, during which the rats reached the true burrow in the Barnes maze when taking polyphenoles (in resveratrol and the food concentrate "Enoant"), and in the control group at the percentage relative to the background indicators, taken as $100 \%$.

Note: significance of differences according to Kraskell-Wallis test at * $\mathrm{p} \leq 0.05$.

According to the number of mistakes, committed to achieve the goal (the true burrow), in the rats, taking the supplement food concentrate "Enoant" and resveratrol, the decrease in the number of mistakes on the $10^{\text {th }}$ day in comparison with the data in the control group average by $50 \%(\mathrm{p}<0,05)$ was registered.

The food concentrate "Enoant" demonstrated the higher effectiveness on the $5^{\text {th }}$ day in comparison with the data of this indicator in the supplement resveratrol. On the $15^{\text {th }}$ day these data were equalized because all the groups of animals were successfully taught.

As the study results show the grape polyphenoles contribute to the increase of nootropic effectiveness because the time, needed to achieve the goal (to find true burrow), decreased and the number of mistakes decreased too in comparison with the data, registered in the control group.

We suppose that grape polyphenoles effect various neurotransmitter systems. 


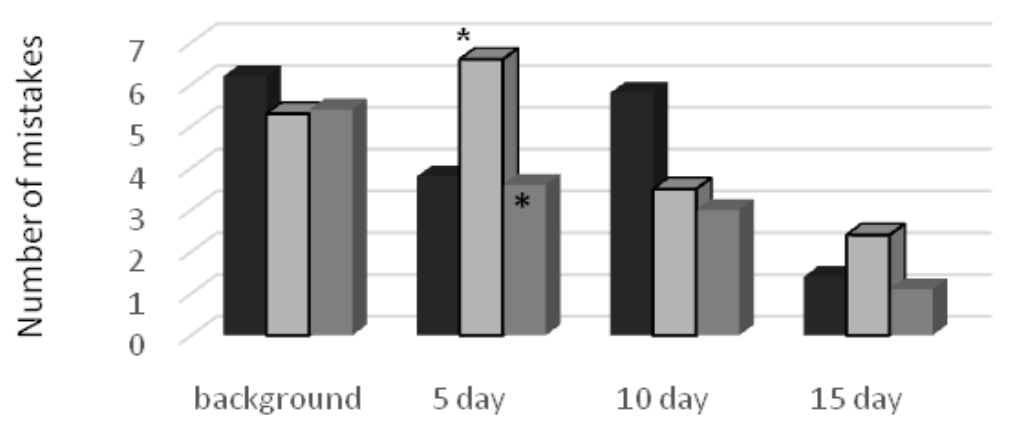

\section{Research Day \\ control aresveratrol a food concentrate "Enoant"}

Fig.2. Changes in the number of mistakes, made by the animals to achieve the goal (the true burrow) in the Barnes maze when taking grape polyphenoles and in the control group.

Notes: significance of differences according to Kraskell-Wallis test at * $\mathrm{p} \leq 0.05$.

Resveratrol promotes the increase of physical activity. The rats, that took the supplement resveratrol, demonstrated a notable increase in the velocity and the time of searching the true burrow, but a decrease in the number of mistakes, that animals made before achieving the goal. According to the scientific literature review and our own observation, we suppose, that resveratrol gets involved in monoaminergic transmission (dopamine). So, the scientific literature data evidence that resveratrol influences the activity of the central and peripheral dopamine. Resveratrol as sirtuin activator [5] regulates the transmission of dopamine [6-8].

Liberto V. Di observed a resveratrol-induced increase in dopamine transporter levels in the striatum of the dopaminergic cells in culture [7]. At the same time, the content of dopamine in the synaptic cleft increases. Probably, this is the reason for the observed behavior and strategies under the influence of resveratrol in the Barnes maze. But, this circumstance requires the involvement of studies using dopamine receptor blockers.

The food concentrate "Enoant" demonstrates a more nootropic activity, because the animals that took the food concentrate made fewer mistakes. Moreover, these animals were more passive in the Barnes maze. It may be due to the fact that food concentrate Enoant shows great affinity to GABA-dependent processes. There are monomeric flavonoids and non-flavonoids as well as anthocyanins in the content of "Enoant" [3, 9].

Epicatechin gallate (one of monomeric flavonoids), that "Enoant" contains, exhibits nootropic action. In the researches of Kristy $\mathrm{Lu} \mathrm{[10]} \mathrm{it} \mathrm{is} \mathrm{mentioned} \mathrm{that} \mathrm{epicatechin} \mathrm{gallate}$ partially or completely suppresses negative modulation of GABA (A) receptors, this stabilizes mood and reduces anxiety. But this requires an additional research

\section{Conclusion}

1. The intake of grape polyphenoles contributes to the change in the nootropic activity in the Barnes maze, as it is evidenced by the dynamics of the number of mistakes and by the time to reach the goal (the true burrow).

2. Grape polyphenoles contribute to the reduction of the number of mistakes in the Barnes maze in comparison with the control group: on the 10th day in the group, taking the food 
concentrate ""Enoant"", by $48 \%(\mathrm{p} \leq 0.05)$ and in the group, taking the drug "Resveratrol", by $39 \%(\mathrm{p} \leq 0.05)$.

3. Grape polyphenoles help to reduce the time spent by animals on the search for the true burrow during the complicated modification in the Barnes maze earlier in comparison with the control group: on the 5th day in the group that took the food concentrate "Enoant" $21.0 \%(\mathrm{p} \leq 0.05)$ and in the group taking the supplement resveratrol by $48 \%(\mathrm{p} \leq 0.05)$.

\section{References}

1. M. Kodali, V. K. Parihar, B. Hattiangady et al., Sci. Rep., 5, 8075 (2015)

2. Yu. A. Ohay, Magarach. Viticulture and Winemaking, 1, 37 (2000).

3. Yu. A. Ohay, Magarach. Viticulture and Winemaking, 1, 37 (2003).

4. V. M. Semonov, A. M. Yarosh, Ukrainskiy biokhimicheskiy zhurnal, 57, 50 (1985).

5. C. Canto, J. Auwerx, Pharmacol., 64, 166 (2012).

6. T. Shuto, M. Kuroiwa, Y. Koga, Neurosci. Lett., 522, 107 (2013).

7. V. DiLiberto, J. Makela, L. Korhonen, Neuropharmacology, 62, 1011 (2012).

8. Y. Xu, Z. Wang, W. You, Eur. Neuropsychopharmacol., 20, 405 (2010).

9. M. T. Bardo, Crit. Rev. Neurobiol., 12, 37 (1998).

10. K. Lu, M.A. Gray, C. Oliver, Hum. Psychopharmacol., 19, 457 (2004). 